\title{
Documento de consenso
}

\section{Documento de consenso sobre el abordaje de la enfermedad de Chagas en Atención Primaria de salud de áreas no endémicas}

\author{
C. Roca Saumell ${ }^{a}$, A. Soriano Arandes ${ }^{b}$, L. Solsona Díaz ${ }^{c}$, J. Gascón Brustenga ${ }^{d}$, \\ Grupo de consenso Chagas-APS
}

Publicado en Internet:

23-marzo-2015

Carme Roca Saumell: croca.bcn.ics@gencat.cat

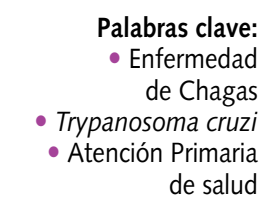

aMédico de Familia. EAP el Clot. Barcelona. Institut Català de la Salut. Universitat de Barcelona. España.

Comissió de Cooperació i Salut Internacional (Cocoopsi). Societat Catalana de Medicina Familiar i

Comunitària (CAMFiC). Sociedad Española de Medicina Tropical y Salud Internacional (SEMTSI) • b Pediatra.

Unitat de Salut Internacional/Tuberculosi Drassanes-Vall Hebron, PROSICS. Barcelona. España.

Asociación Española de Pediatría de Atención Primaria (AEPap). Grup d'Atenció Primària de la Societat

Catalana de Pediatría. Sociedad Española de Medicina Tropical y Salud Internacional (SEMTSI) • `Médico

de Familia. EAP Florida Nord. L’Hospitalet de Llobregat, Barcelona. España. Institut Català de la Salut.

Comissió de Cooperació i Salut Internacional (Cocoopsi). Societat Catalana de Medicina Familiar i Comunitària (CAMFiC). Sociedad Española de Medicina Tropical y Salud Internacional (SEMTSI) • ${ }^{\mathrm{d} C e n t r e}$

de Recerca en Salut Internacional de Barcelona (CRESIB). Iniciativa de Chagas del Institut de Salut Global de Barcelona (ISCLOBAL). Sociedad Española de Medicina Tropical y Salud Internacional (SEMTSI)

La tripanosomiasis americana o enfermedad de Chagas es una enfermedad infecciosa endémica en América Latina continental, causada por el protozoo Trypanosomacruzi. En las últimas décadas, debido a los movimientos poblacionales, se ha expandido más allá de las zonas endémicas, siendo España el país europeo con más inmigrantes latinoamericanos.

Durante años puede permanecer asintomática, pero cuando se manifiesta clínicamente puede ser grave (miocardiopatía dilatada, megacolon, megaesófago). Así como, debido a su transmisión vertical, la detección en embarazadas es una alta prioridad.

Se han elaborado guías de detección de Trypanosomacruzi en circunstancias específicas (bancos de sangre, maternidades, coinfección con $\mathrm{VIH}$, trasplante de órganos); pero detectamos falta de información dirigida a los profesionales de atención primaria. Para facilitar la detección y manejo de esta enfermedad se consideró la necesidad de realizar este documento, redactado y consensuado por médicos de familia, pediatras de Atención Primaria y especialistas en Salud Internacional.

\section{Consensus document for the detection and management of Chagas disease in Primary Health Care in non-endemic areas}

Chagas disease is caused by the protozoan Trypanosoma cruzi. Although it is commonly transmitted by an insect vector in continental Latin-America, in recent decades, due migration, it has been diagnosed in other countries such as Spain, the European country with a largest immigrant population of Latin Americans. For a long time, the patient remains asymptomatic, but some years after this stage, the symptoms can be serious (dilated cardiomyopathy, megacolon, megaesophagus). In addition, detection in pregnant women has a high priority because of the route of vertical transmission.

Key words: Several specific guidelines about Chagas disease have been developed (blood banks, maternal hospitals, HIV co-infection, organ transplant). But lack of information to primary care professionals has been detected. We consider this document written and agreed by family physicians, pediatricians and spe-

- Chagas disease

- Trypanosoma cruzi - Primary Health Care

Documento publicado simultáneamente en la revista Atención Primaria (Sociedad Española de Medicina de Familia y Comunitaria).

Cómo citar este artículo: Roca Saumell C, Soriano-Arandes A, Solsona Díaz L, Gascón Brustenga J, Grupo de consenso Chagas-APS. Documento de consenso sobre el abordaje de la enfermedad de Chagas en Atención Primaria de salud de áreas no endémicas. Rev Pediatr Aten Primaria. 2015;17:e1-e12. 


\section{INTRODUCCIÓN}

\section{Antecedentes epidemiológicos}

La tripanosomiasis americana o enfermedad de Chagas (EC) es una enfermedad infecciosa endémica en 21 países de América Latina (Tabla 1), estrechamente vinculada con aspectos socio-económico-culturales deficitarios, siendo reconocida por la Organización Mundial de la Salud como una de las diecisiete enfermedades tropicales desatendidas del mundo.

Está causada por la infección de un parásito protozoario hemoflagelado, Trypanosoma cruzi (T. cruzi).

Su principal vía de transmisión en área endémica es vectorial, a partir de insectos triatominos que tienen su ecosistema natural en el continente americano; estos insectos son conocidos como "vinchuca" en Bolivia, Argentina y Chile, "chipo" en Venezuela, "chiribico" en Colombia, "chinche besucona" en México o "barbeiro" en Brasil entre otros. Si bien su hábitat natural se extiende desde el sur de Estados Unidos a la región central de Argentina y Chile, su distribución es muy heterogénea, siendo las áreas más afectadas el centro y sur de Bolivia (con prevalencias cercanas al 30\% de la población general), norte de Argentina, sur de Perú y determinadas áreas de Paraguay, Brasil, Ecuador, Nicaragua, El Salvador y sur de México.

Otras formas de transmisión son: congénita, por transfusión de sangre, por trasplante de órganos, vía oral (por la ingesta de alimentos contaminados con parásitos) o por accidente de laboratorio.

En las últimas décadas, debido a los flujos migratorios, se han producido importantes cambios epidemiológicos en la EC, diagnosticándose un número creciente de personas en áreas no endémicas principalmente en Europa y Norteamérica. En Europa, el estado Español es el país más afectado y se estima que puede haber entre 50 y 70000 personas con $\mathrm{EC}^{1}$.

Son numerosas las publicaciones que han detectado casos individuales o que han evaluado la prevalencia de la EC en nuestro país, tanto en la población inmigrante ${ }^{2-4}$ como en los bancos de sangre ${ }^{5}$, en los programas de trasplante ${ }^{6}$ y maternidades ${ }^{7-9}$. En España estos resultados, junto a estudios de evaluación económica ${ }^{10}$, sirvieron para mostrar la conveniencia de implementar protocolos de actuación para el control de las vías de transmisión del $T$. cruzi posibles en área no endémica.

El Real Decreto 1088/2005 establece la obligatoriedad de cribar en los bancos de sangre a los potenciales donantes con riesgo epidemiológico de EC. EI Plan nacional de sangre de cordón (14/03/2008) recoge las mismas recomendaciones que las relacionadas con la transfusión de sangre.

La transmisión vertical se controla por el cribado sistemático de gestantes. En Valencia ${ }^{11}$, Catalunya ${ }^{12}$ y Galicia existen protocolos específicos para el cribado y diagnóstico de EC en mujeres embarazadas latinoamericanas y en sus recién nacidos.

En el año 2005 la Sociedad Española de Medicina Tropical y Salud Internacional inició la elaboración de documentos de consenso para el diagnóstico y tratamiento de la EC importada, que abarcan diversos aspectos de la problemática y complicaciones de esta enfermedad ${ }^{13-17}$.

Tabla 1. Países donde existe enfermedad de Chagas endémica

\begin{tabular}{|l|l|l|}
\hline Argentina & Ecuador & Nicaragua \\
\hline Belice & El Salvador & Panamà \\
\hline Bolivia & Guatemala & Paraguay \\
\hline Brasil & Guayana & Perú \\
\hline Chile* & Guayana Francesa & Surinam \\
\hline Colombia & Honduras & Uruguay* \\
\hline Costa Rica & México & Venezuela \\
\hline
\end{tabular}

*Eliminación de la transmisión vectorial, pero antiguos pacientes afectados. 


\section{Justificación}

A pesar de los avances descritos, se estima que el porcentaje de población infectada por T. cruzi diagnosticada en Europa no alcanza el $10 \%$ del total ${ }^{18}$. Existen aún importantes retos para el sistema sanitario en el abordaje de la EC, como son: extender la implementación del programa de control de transmisión vertical a todas las comunidades y el diagnóstico, tratamiento y seguimiento adecuado de los infectados.

La Atención Primaria de Salud (APS) constituye el marco habitual de acceso al sistema de salud y es el ámbito idóneo para detectar de forma precoz aquellas personas afectadas por la EC que pudieran beneficiarse de un tratamiento.

\section{Objetivos del documento}

Definir las características demográficas, epidemiológicas y clínicas de las personas susceptibles de estar infectadas por T. cruzi, para facilitar su detección y manejo por el médico de familia o pediatra, integrando el cuidado de los afectados por la EC en los servicios de APS.

\section{Metodología}

Para la elaboración del documento se realizó una revisión exhaustiva de la evidencia científica disponible utilizando el motor de búsqueda PubMed de libre acceso a la base de datos MEDLINE (última revisión de marzo 2014).

El documento ha sido sometido a la revisión externa de un grupo multidisciplinar de profesionales expertos miembros de diversas sociedades científicas, que han participado de forma voluntaria y altruista.

\section{ASPECTOS CLÍNICOS DE LA ENFERMEDAD DE CHAGAS}

\section{Fases de la enfermedad de Chagas y manifestaciones clínicas}

La EC evoluciona en dos fases, aguda y crónica, cada una de ellas con características clínicas, criterios diagnósticos y terapéuticos diferentes.

\section{Fase aguda}

Se inicia al adquirir la infección por cualquiera de sus vías y dura entre 30 y 90 días. Se caracteriza por la presencia de una alta parasitemia detectable por métodos parasitológicos directos. Generalmente es asintomática o presenta síntomas inespecíficos como fiebre, adenomegalias, hepatoesplenomegalia, anemia, anorexia o diarrea.

En áreas endémicas, cuando la vía de transmisión es vectorial, en un $8 \%$ de los casos pueden aparecer síntomas específicos como el chagoma de inoculación. Las manifestaciones clínicas graves y poco frecuentes de esta fase son miocarditis y meningoencefalitis.

En países no endémicos, la fase aguda solo se puede observar en neonatos (transmisión congénita), personas transfundidas o trasplantadas.

La mayoría de los recién nacidos con EC adquirida por transmisión congénita permanecen asintomáticos. Cuando aparecen, las manifestaciones y cuadros clínicos más importantes son hepatoesplenomegalia, hepatitis, sepsis, meningitis, miocarditis o anemia hemolítica, pudiendo ser ocasionalmente causa de muerte.

Habitualmente, las manifestaciones agudas de la EC remiten de forma espontánea, dando paso a la fase crónica indeterminada o asintomática.

\section{Fase crónica}

La parasitemia periférica es escasa e intermitente y el diagnóstico se realiza por medio de técnicas serológicas.

EC en fase crónica, forma indeterminada, asintomática, consiste en la positividad de las pruebas serológicas con ausencia de afectación orgánica demostrable y puede persistir toda la vida del paciente.

Aproximadamente un 20-30\% de estos pacientes, entre 10 y 20 años después de la primoinfección, evolucionan lentamente hacia una fase crónica sintomática que cursa con disfunción autonómica, microinflamaciones y fibrosis, produciendo lesiones irreversibles en los órganos diana (corazón, esófago, colon y sistema nervioso periférico). 
EC en fase crónica con afectación cardiaca. Cursa con miocardiopatía dilatada y trastornos de la conducción, que producirá insuficiencia cardiaca, tromboembolismos y arritmias.

Las alteraciones electrocardiográficas y ecocardiográficas más frecuentes en la cardiopatía chagási$\mathrm{ca}^{16,19}$ se detallan en la Tabla 2.

EC en fase crónica con afectación digestiva. Puede llegar a afectar al 15-20\% de los pacientes con EC. Se producen dilataciones del tracto digestivo (megaesófago, megacolon) y trastornos motores gastrointestinales como acalasia esofágica, alteración del vaciado gástrico, alteración del tránsito intestinal y estasis biliar.

Los síntomas principales son: disfagia, odinofagia, regurgitación, estreñimiento crónico y/o dolor abdominal.

\section{EC en fase crónica con afectación mixta cardio-di- gestiva.}

EC en fase crónica con afectación neurológica. La patologia cerebrovascular puede ser secundaria a la cardiopatía chagásica (insuficiencia cardiaca, trombosis mural y/o arritmias) pero también puede ser el primer signo de la EC en pacientes asintomáticos o con disfunción sistólica leve, por lo que los pacientes con patología cerebrovascular que provienen de regiones endémicas deben ser examinados para la infección por T. cruzi ${ }^{20}$.
EC crónica reactivada ${ }^{21}$. Los pacientes con inmunosupresión pueden presentar una reactivación de la EC. En pacientes con infección por VIH, las alteraciones del sistema nervioso central pasan a ser predominantes y a veces exclusivas. En pacientes inmunodeprimidos por leucemias y terapias inmunosupresoras, destacan los cuadros de miocarditis y de meningoencefalitis. En los pacientes trasplantados son frecuentes los nódulos subcutáneos.

\section{DIAGNÓSTICO}

Solo es posible detectar la forma circulante del T. cruzi durante la fase aguda de la infección, por lo que los métodos diagnósticos más idóneos son los directos (parasitológicos) y los moleculares (PCR). Estas pruebas también son útiles en los casos de reactivación.

En la fase crónica de la enfermedad, la parasitemia es de baja intensidad e intermitente, por lo que el diagnóstico se realiza mediante la detección de anticuerpos inmunoglobulina G (IgG) anti-T. cruzi (diagnóstico serológico).

De acuerdo con los criterios internacionales, se consideran positivos para EC, aquellos sueros que son reactivos mediante dos técnicas serológicas que utilizan antígenos distintos y cuando los resultados son discordantes o dudosos es necesario realizar una tercera prueba confirmatoria.

Tabla 2. Alteraciones electrocardiográficas y ecocardiográficas más frecuentes en la cardiopatía chagásica

\begin{tabular}{|l|l|}
\hline $\begin{array}{l}\text { Alteraciones } \\
\text { electrocardiográficas }\end{array}$ & Bloqueo completo de la rama derecha aislado o asociado a hemibloqueo anterior de rama \\
izquierda \\
Extrasístoles ventriculares, aisladas o repetitivas \\
Alteración primaria de la repolarización ventricular, que puede simular cardiopatía isquémica. \\
Zonas eléctricamente inactivas (ondas q) \\
Bloqueos auriculoventriculares \\
Otras alteraciones menos frecuentes: bloqueo de rama izquierda, disfunción sinusal, \\
taquiarritmia supraventricular (fibrilación auricular)
\end{tabular}


En los recién nacidos de madres con EC, las pruebas serológicas no son útiles para llegar a un diagnóstico precoz de la EC congénita, ya que las IgG anti-T. cruzi halladas en el recien nacido hasta los nueve meses de vida pueden ser de origen materno y la detección de IgM no ofrece resultados satisfactorios. Por lo que, siempre que sea posible, deben realizarse pruebas parasitológicas directas (microhematocrito o PCR) durante el primer mes de vida para detectar el parásito o el ADN del mis$\mathrm{mo}^{22}$ y reservar las pruebas serológicas a partir de los nueve meses de edad.

\section{TRATAMIENTO}

En la fase aguda de la EC y en niños menores de 12 meses, se establecen cifras serológicas de curación cercanas al 100\% tras el tratamiento con benznidazol (BNZ) ${ }^{23}$. En la fase crónica de la enfermedad, las tasas de curación disminuyen. Aunque la patogénesis de la EC crónica es multifactorial, la persistencia del parásito es el hecho clave para su desarrollo ${ }^{24}$. Por ello, la eliminación o la disminución de la carga parasitaria modifican la evolución natural de la enfermedad ${ }^{25}$.

Actualmente los únicos fármacos disponibles para tratar la EC son nifurtimox (NF) y BNZ. Ambos fármacos están contraindicados en mujeres embarazadas y en pacientes con insuficiencia renal o hepática severa. Durante el tratamiento debe evitarse el consumo de alcohol.

La pauta terapéutica consiste en:

- BNZ: en adultos $5 \mathrm{mg} / \mathrm{kg}$ de peso/día, (máximo $300 \mathrm{mg} / 24 \mathrm{~h}$ ), repartidos en 2-3 dosis orales diarias, durante 60 días. En niños menores 12 años, la dosis recomendada es de $10 \mathrm{mg} / \mathrm{kg}$ de peso/día. BNZ puede producir efectos secundarios gastrointestinales al inicio del tratamiento, pero suelen ser leves y bien tolerados. Las reacciones adversas que más frecuentemente inducen al abandono del tratamiento son las derivadas de hipersensibilidad cutánea, con aparición de exantema eritematoso y pruriginoso que en ocasiones puede ser grave.
- NF (no disponible en España): en adultos 8-10 $\mathrm{mg} / \mathrm{kg} / \mathrm{día}$, administrado oralmente en 3 o 4 tomas diarias, durante 60 días. En niños de 11 a 16 años la dosis recomendada es de 12,5-15 $\mathrm{mg} / \mathrm{kg} /$ día y en niños menores de 11 años la dosis será de 15-20 mg/kg/día.

Las reacciones adversas más frecuentes son la anorexia, náuseas, vómitos y en menor medida, dolor abdominal, diarrea, pérdida de peso, irritabilidad, somnolencia y alteraciones psiquiátricas. La neuropatía periférica es un efecto dosis dependiente, que ocurre más frecuentemente durante el 2... mes de tratamiento y que requiere la discontinuidad del mismo.

En la Tabla 3 se exponen las recomendaciones para el tratamiento antiparasitario de la EC, según el nivel de evidencia.

\section{ABORDAJE DE LA ENFERMEDAD DE CHAGAS DESDE LA ATENCIÓN PRIMARIA DE SALUD}

\section{Anamnesis específica en relación a la EC}

Al ser una enfermedad parasitaria crónica que puede permanecer asintomática, el diagnóstico requiere de un alto grado de sospecha clínica, basado en los antecedentes epidemiológicos del paciente (Figuras 1 y 2 ).

El objetivo de la anamnesis será doble:

- Evaluar el riesgo epidemiológico de la infección por T. cruzi. Valorar el origen del paciente o la estancia prolongada (más de un mes) en áreas endémicas y recoger información sobre:

- País de origen, departamento o región y/o municipio.

- Residencia en zonas rurales o urbanas, haber habitado en casas de adobe.

- Conocimiento de la existencia de la EC en la área o áreas donde ha vivido ${ }^{26}$, o de algún familiar con EC, preguntando específicamente por el antecedente materno.

- Si ha recibido transfusiones de sangre o ha sido receptor de transplantes en el país endémico. 
Tabla 3. Recomendaciones para el tratamiento antiparasitario de la EC, según el nivel de evidencia

Tratamiento farmacológico de la EC
Fuerza y calidad de la recomendación ${ }^{a}$

Adaptadas de Bern et al. ${ }^{27}$ y Sosa-Estani et al. de Bern

\section{Siempre debe ofrecerse en: \\ Infección aguda por T. cruzi \\ Infección congénita inicial por T. cruzi \\ Niños $\leq 12$ a nos con infección crónica por T. cruzi \\ Niños de 13-18 años con infección crónica por T. cruzi-Reactivación de T. \\ cruzi en pacientes $\mathrm{VIH} /$ sida u otras inmunosupresiones}

\section{Por lo general debe ofrecerse a:}

Mujeres en edad fértil

Adultos de 19-50 años con forma indeterminada o con cardiopatía leve o moderada

Pacientes si tratamiento inminente con inmunosupresores

Tratamiento opcional en:

Adultos $>50$ años y sin cardiopatía avanzada

Pacientes con EC gastrointestinal, pero sin cardiopatía avanzada

En general no se debe ofrecer tratamiento en:

Miocardiopatía chagásica avanzada con insuficiencia cardiaca congestiva

Megaesófago con deterioro significativo de la deglución

Nunca se debe ofrecer tratamiento:

Durante el embarazo

Insuficiecia renal y/o hepática grave

\begin{tabular}{|l|l} 
Al & \\
\hline All & Al \\
\hline All & $\mathrm{BI}$ \\
\hline All & $\mathrm{BI}$ \\
All & \\
\hline
\end{tabular}

\begin{tabular}{|c|c|}
\hline BIII & CII \\
\hline BII & CII \\
\hline
\end{tabular}

CIII

CIII

\begin{tabular}{l|l} 
DIII & CII \\
\hline DIII & \\
\hline & \\
\hline EIII & \\
EIII &
\end{tabular}

DIII

a La clasificación de la fuerza y la calidad de las recomendaciones se ha realizado aplicando una adaptación del sistema utilizado por la Sociedad Americana de Enfermedades Infecciosas (IDSA), tal y como se refleja a continuación:

Fuerza de la recomendación:

A: evidencias muy sólidas para apoyar el uso de una recomendación; sería ofrecida siempre.

B: moderada evidencia para apoyar el uso de una recomendación; generalmente sería ofrecida.

C: escasa evidencia para apoyar una recomendación; opcional.

D: moderada evidencia en contra de una recomendación; generalmente no sería ofrecida.

E: evidencia muy sólida en contra de una recomendación; nunca sería ofrecida.

Calidad de la recomendación:

I: evidencia procedente de al menos un ensayo clínico aleatorizado.

II: evidencia procedente de al menos un ensayo no aleatorizado, bien diseñado, bien de estudios de cohortes, de estudios analíticos de casos y controles (preferiblemente de más de un centro), de series temporales o de resultados concluyentes obtenidos en estudios experimentales no controlados.

III: evidencia de opiniones de expertos basadas en la experiencia clínica o en estudios descriptivos

Fuentes: Sosa-Estani S, Colantonio L, Segura EL. Therapy of Chagas disease: Implications fr levels of prevention. J Trop Med. 2012;2012:292138. Bern C. Chagas disease: Management of acute disease, early chronic disease, and disease in immunocompromised hosts. En: UpToDate [en línea]. Disponible en http://goo.gl/iMrAoB

- En la consulta pediátrica, aparte de saber si el niño es originario de zona endémica (considerar los niños adoptados), es importante detectar a los nacidos en España hijos de madre originaria de zona endémica y comprobar si se ha realizado el cribado de la EC durante el embarazo y el resultado del mismo.

- Detectar síntomas de probable afectación orgánica. Debe tenerse en cuenta que la mayor parte de los pacientes con EC se encontrarán en fase crónica indeterminada y por consiguiente asintomáticos:

- Para detectar cardiopatía subyacente se interrogará sobre la presencia de síntomas secundarios a alteraciones del rítmo cardiaco (palpitaciones, síncopes), insuficiencia cardiaca, fenómenos tromboembólicos venosos y sistémicos (embolias pulmonares o sistémicas, patología vascular cerebral generalmente isquémica) o alteraciones microvasculares (dolor precordial). 
Figura 1. Algoritmo para el abordaje de la enfermedad de Chagas en población adulta en Atención Primaria de Salud en España.

En todos los pacientes adultos originarios de Latinoamérica continental hay que realizar anamnesis y exploración física si:

- Antecedentes epidemiológicos sugestivos (haber residido en zona rural, en casas de adobe, conocer

la EC en el país de origen, haber recibido transfusiones en zona endémica).

- Embarazadas.

- Inmunodeprimidos

- Con síntomas sugerentes de EC.

Estudio familiar y del entorno con factores de riesgo
Serología Trypanosoma cruzi
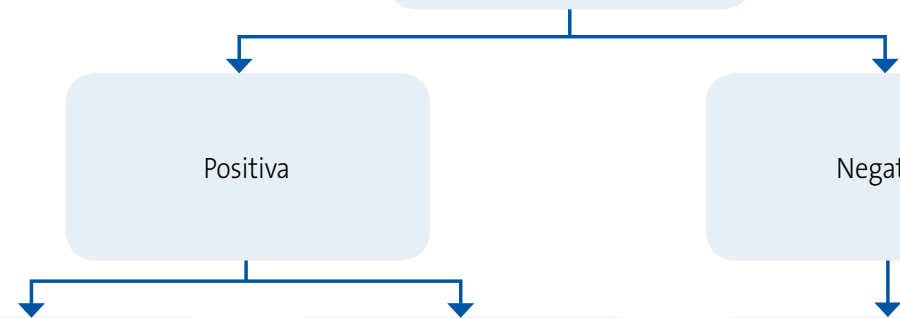

ECG

Rx tórax

Ecocardiografía

Sintomas digestivos

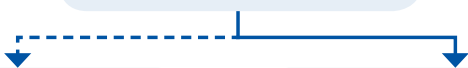

No enfermedad de Chagas
Derivación a unidad de salud internacional para completar estudio y tratamiento
EC crónica indeterminada

EC con afectación cardiaca

EC con afectación digestiva

EC con afectación mixta

EC: enfermedad de Chagas; ECG: electrocardiograma; Rx: radiografía.

- Para detectar afectación digestiva se interrogará sobre ${ }^{17}$ síntomas relacionados con alteracion esofágica (disfagia, regurgitación, odinofagia, tos nocturna, sialorrea, hipertrofia parotídea), con alteración gástroduodenal (dispepsia, pirosis, distensión abdominal, epigastralgia), y con alteración colónica (estreñimiento, cambio del ritmo deposicional, sensación de evacuación incompleta).

\section{Exploración física específica de la enfermedad de Chagas}

- Peso, talla (o longitud en menores de dos años) e índice de masa corporal (IMC) en $\mathrm{kg} / \mathrm{m}^{2}$.

- Presión arterial, frecuencia cardiaca.

- Exploración cardiovascular detallada. Auscultación cardiaca durante un minuto.

- Exploración abdominal exhaustiva. 
Figura 2. Algoritmo para el abordaje de la enfermedad de Chagas en población pediátrica en atención primaria de salud en España

Pacientes de 0 a 14 años atendidos en un centro de APS

Revisar antecedentes epidemiológicos:

- Haber nacido en Latinoamérica continental.

- Haber nacido en área no endémica para EC pero haber vivido durante un periodo superior a un mes en algún país de Latinoamérica continental.

- Hijos de una madre nacida en algún país de Latinoamérica continental.

- Haber recibido alguna transfusión de hemoderivados en algún país de Latinoamérica continental.

- Haber sido receptores de algún trasplante de un órgano en algún país de Latinoamérica continental.

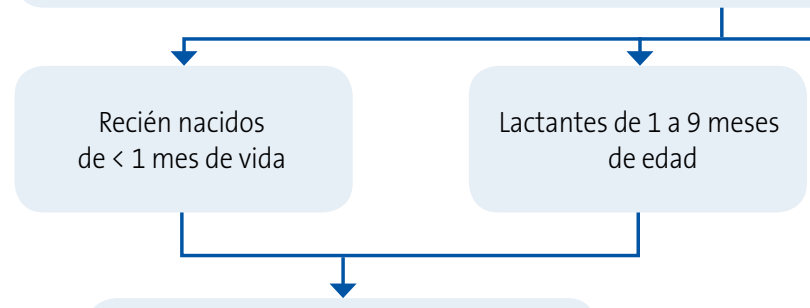

Revisar carné de embarazada y averiguar si la madre fue cribada para EC durante el embarazo

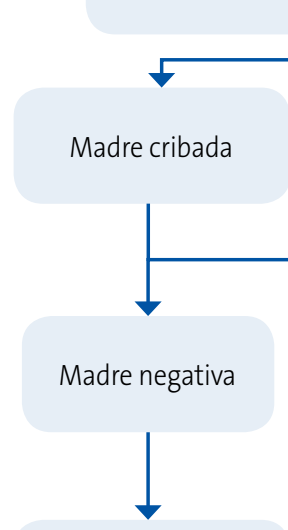

Fin del seguimiento

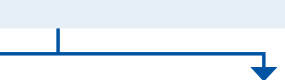


Población susceptible de estudio

\section{Población adulta}

En los países no endémicos para EC, tras los resultados obtenidos en diversos estudios ${ }^{4,27}$, se recomienda la detección de la infección por T. cruzi a los adultos originarios de Latinoamérica continental o que hayan realizado largas estancias (más de un mes) en estos países, que hayan sido informados correctamente y accedan a ser estudiados y que presenten:

- Antecedentes epidemiológicos sugestivos.

- Embarazadas.

- Inmunodeprimidos.

- Síntomas/signos sugerentes de EC.

\section{Población pediátrica}

Los pediatras de APS tienen una responsabilidad fundamental para la detección y abordaje de la EC en la población que atienden ${ }^{28}$. Todo niño que es atendido en una primera visita al centro es tributario de ser interrogado por su origen biográfico y familiar.

Serán susceptibles de ser estudiados los niños en edad pediátrica (de 0 a 14 años), previa información y consentimiento de los padres o tutores, con los siguientes antecedentes epidemiológicos:

- Haber nacido o haber realizado largas estancias (más de un mes) en Latinoamérica continental.

- Ser hijos de madre nacida en país endémico.

- Haber recibido alguna transfusión de hemoderivados o haber sido receptores de algún trasplante de un órgano en país endémico.

\section{Métodos diagnósticos}

Para descartar la EC en fase crónica desde la APS deben solicitarse anticuerpos IgG anti-T. cruzi.

\section{Exploraciones complementarias}

Con el objetivo de determinar si un paciente con infección por T. cruzi presenta patología orgánica, se recomienda practicar:
- Electrocardiograma (ECG) convencional de 12 derivaciones, con un registro largo de 30 segundos, en DII.

- Radiografía de tórax (posteroanterior y lateral). La evidencia de cardiomegalia podría ser un factor predictivo de riesgo de muerte súbita en pacientes con EC crónica. También puede detectar alteraciones del mediastino secundarias a megaesófago.

- Ecocardiografía: valorar la función ventriculary descartar aneurismas.

En la Tabla 4 se detallan otras exploraciones complementarias optativas en función de la disponibilidad y de la clínica.

\section{TRATAMIENTO}

A pesar de que BNZ es el único fármaco autorizado en España para el tratamiento de la EC, no está disponible en el circuito habitual de las farmacias y solo puede dispensarse como medicamento extranjero.

Durante el tratamiento, es aconsejable realizar visitas de seguimiento y control analítico (hemograma, la función hepática y renal) cada 2-3 semanas para controlar la aparición de reacciones adversas y el cumplimiento terapéutico. La coordinación entre el servicio especializado y la APS puede ofrecer grandes ventajas.

En mujeres en edad fértil descartar la posibilidad de embarazo previo al inicio del tratamiento e indicar la anticoncepción durante el mismo.

\section{Seguimiento}

\section{Enfermedad de Chagas en fase crónica indeterminada}

Para detectar precozmente la afectación orgánica se aconseja practicar anualmente un ECG y realizar anamnesis sobre síntomas y signos de afectación cardiaca o digestiva. 


\begin{tabular}{|c|c|}
\hline $\begin{array}{l}\text { Para completar el estudio } \\
\text { cardiológico (dependiendo de la } \\
\text { clínica que presente el paciente) }\end{array}$ & $\begin{array}{l}\text { Estudio Holter de } 24 \text { horas: permitirá establecer la posible relación de los síntomas con una } \\
\text { arritmia, identificar a los pacientes en riesgo de muerte súbita y evaluar la presencia de } \\
\text { disfunción autonómica } \\
\text { Estudio electrofisiológico } \\
\text { Prueba de esfuerzo } \\
\text { Coronariografía } \\
\text { Biopsia miocárdica }\end{array}$ \\
\hline $\begin{array}{l}\text { Para completar el estudio } \\
\text { digestivo (dependiendo de la } \\
\text { clínica que presente el paciente) }\end{array}$ & $\begin{array}{l}\text { Estudio radiológico con enema opaco, teniendo en cuenta las técnicas de Ximenes para la } \\
\text { realización de estudio radiológico baritado en pacientes con sospecha de afectación digestiva } \\
\text { chagásica } \\
\text { Esofagograma, teniendo en cuenta las técnicas de Rezende para la realización de estudio } \\
\text { radiológico baritado en pacientes con sospecha de afectación digestiva chagásica } \\
\text { Diagnóstico de la infección por Helicobacter pylori: a todos los pacientes que presenten } \\
\text { dispepsia, pirosis o signos de distensión abdominal postprandial. En los pacientes > } 50 \\
\text { años el estudio debe ser endoscópico mientras que en < } 50 \text { años puede realizarse mediante } \\
\text { técnicas no invasivas (habitualmente test del aliento: estrategia test and treat) } \\
\text { En caso de Helicobacter pylori positivo se recomienda el tratamiento erradicador y } \\
\text { posteriormente confirmar su erradicación } \\
\text { Estudio de parásitos en heces: síntomas digestivos atribuidos a la EC pueden estar } \\
\text { producidos por parasitosis intestinales. Se recomienda estudio seriado de parásitos en heces } \\
\text { mediante tres muestras de diferentes días, incluyendo un análisis en fresco } \\
\text { Manometría esofágica: a todos los pacientes que aún con esofagograma normal presenen } \\
\text { sintomatología esofágica } \\
\text { Estudios endoscópicos: siempre que se constate megaesófago para valorar el estado de la } \\
\text { mucosa esofágica. Y siempre que se sospeche de otra patología digestiva por el cuadro clínico } \\
\text { o los antecedentes familiares que refiera el paciente }\end{array}$ \\
\hline
\end{tabular}

EC: enfermedad de Chagas.

\section{Enfermedad de Chagas con afectación cardiaca o digestiva o mixta}

El seguimiento de la patología cardiaca y/o digestiva chagásica, por el médico de familia, será la habitual que realizará en estas mismas patologías debidas a otras etiologías.

\section{Recien nacidos hijos de madres positivas}

Aunque el resultado de la parasitemia haya sido negativo en el periodo neonatal, es obligatorio realizar un seguimiento del niño y una serología a los 9-12 meses de edad, cuando es esperable que los anticuerpos maternos hayan sido eliminados. Una serología negativa a esta edad descarta la transmisión vertical de la EC. Si es claramente positiva, con un título serológico elevado, se iniciará el tratamiento del lactante, pero si el título está cercano al punto de corte deberá confirmarse el diagnóstico con una nueva determinación serológica un mes después.
No se ha demostrado la presencia de T. cruzi en la leche de las madres con EC, por lo que la lactancia materna no se desaconseja a no ser que existan heridas sangrantes en el pezón ${ }^{24}$.

\section{ASPECTOS PREVENTIVOS EN VIAJEROS}

La APS ocupa una situación clave para la prevención primaria de la EC, aconsejando medidas antivector a los viajeros a zonas endémicas (especialmente el altiplano andino y Bolivia).

La población de mayor riesgo la constituyen los inmigrantes que viajan a zona endémica para visitar a familiares y amigos.

El riesgo de contraer la EC durante el viaje es bajo, aunque hay que considerar factores como el tipo de viaje, la duración, la estancia en zonas rurales y el tipo de alojamiento.

Debe aconsejarse a toda persona que haya estado en zona endémica de EC y que al regreso de su viaje 
presente síntomas de malestar, fiebre, erupción cutánea, dolor abdominal o cualquier otro síntoma que acuda a su médico.

\section{OTRAS CONSIDERACIONES}

La APS será responsable del estudio de los familiares $u$ otras personas del entorno del paciente con EC que hayan sido expuestas a los mismos riesgos epidemiológicos.

\section{BIBLIOGRAFİA}

1. Gascón J, Bern C, Pinazo MJ. Chagas disease in Spain, the United States and other non-endemic countries. Acta Trop. 2010; 115:22-7.

2. Muñoz J, Gomez i Prat J, Gallego M, Gimeno F, Trevino B, López-Chejade P, et al. Clinical profile of Trypanosoma cruzi infection in a non-endemic setting: immigration and Chagas disease in Barcelona (Spain). Acta Trop. 2009;111:51-5.

3. Soriano Arandes A, Muñoz Gutierrez J, Vergés Navarro M, Castells Doménech C, Portús Vinyeta M, Gascón Brustenga J. Prevalence of Chagas disease in the Latin American immigrant population in a primary health centre in Barcelona (Spain). Acta Trop. 2009; 112:228-30

4. Roca C, Pinazo MJ, López-Chejade P, Bayó J, Posada E, López-Solana, J, et al. Chagas Disease among the Latin American Adult population attending in a Primary Care Center in Barcelona, Spain. PLoS Negl Trop Dis. 2011;5:e1135.

5. Piron M, Vergés M, Muñoz J, Casamitjana N, Sanz S, Maymó RM, et al. Seroprevalence of Trypanosoma cruzi infection in at-risk blood donors in Catalonia (Spain). Transfusion. 2008;48:1862-8.

6. Forés R, Sanjuán I, Portero F, Ruiz E, Regidor C, LópezVélez $R$, et al. Chagas disease in a recipient of cord blood transplantation. Bone Marrow Transplant. 2007; 39127-8.

7. Munoz J, Portus M, Corachan M, Fumado V, Gascon J. Congenital Trypanosoma cruzi infection in a nonendemic area. Trans R Soc Trop Med Hyg. 2007;101:1161-2.

\section{CONFLICTO DE INTERESES}

Los autores declaran no presentar conflictos de intereses en relación con la preparación y publicación de este artículo.

\section{ABREVIATURAS}

APS: Atención Primaria de Salud • BNZ: benzinidazol • EC: enfermedad de Chagas • ECG: electrocardiograma • IgG: inmunoglobulina G • IMC: índice de masa corporal • NF: nifurtimox.

8. Muñoz J, Coll O, Juncosa T, Verges M, del Pino M, Fumadó V, et al. Prevalence and vertical transmission of Trypanosoma cruzi infection among pregnant Latin American women attending 2 maternity clinics in Barcelona, Spain. Clin Infect Dis. 2009;48:1736-40.

9. Paricio-Talayero JM, Benlloch-Muncharaz MJ, Collardel-Castillo JI, Rubio-Soriano A, Serrat-Pérez C, Magraner-Egea J, et al. Epidemiological surveillance of vertically-transmitted Chagas disease at three maternity hospitals in the Valencian Community. Enferm Infecc Microbiol Clin. 2008;26:609-13.

10. Sicuri E, Muñoz J, Pinazo MJ, Posada E, Sánchez J, Alonso PL, et al. Economic evaluation of Chagas disease screening of pregnant Latin American women and of their infants in a non endemic area. Acta Trop. 2011;118:110-7.

11. Conselleria de Salut, Comunitat Valenciana. Enfermedad de chagas importada. Protocolo de actuación en la Comunitat Valenciana. En: Associació de Comares [en línea] [consultado el 16/03/2015]. Disponible en www.matronas-cv.org/categorias-principales/ documentos/profesionales/i/475/65/enfermedadde-chagas-importada-protocolo-de-actuacion-enla-comunitat-valenciana

12. Departament de Salut, Generalitat de Catalunya. Protocol de cribratge i diagnòstic de malaltia de Chagas en dones embarassades Ilatinoamericanes i en els seus nadons. En: Departament de Salut. Generalitat de Catalunya [en línea] [consultado el 16/03/2015]. Disponible en http://www.gencat.cat/ salut/depsalut/html/ca/dir2384/protchagas2010. pdf 
13. Gascón J. Diagnóstico y tratamiento de la enfermedad de Chagas importada. Med Clin (Barc). 2005; 125:230-35.

14. Pinazo MJ, Miranda B, Rodríguez-Villar C, Altclas J, Brunet Serra M, García-Otero EC, et al. Recommendations for management of Chagas disease in organ and hematopoietic tissue transplantation programs in nonendemic areas. Transplant Rev (Orlando). 2011;25:91-101.

15. Pérez-Molina JA, Rodríguez-Guardado A, Soriano A, Pinazo MJ, Carrillero B, García-Rodríguez $M$, et al. Guidelines on the treatment of chronic coinfection by Trypanosoma cruzi and HIV outside endemic areas. HIV Clin Trials. 2011;12:287-98.

16. Gascón J, Albajar P, Cañas E, Flores M, Gomez i Prat J, Herrera RN, et al. Diagnóstico, manejo y tratamiento de la cardiopatía chagásica crónica en áreas donde la infección por Trypanosoma cruzi no es endémica. Rev Esp Cardiol. 2007;60:285-293.

17. Pinazo MJ, Cañas E, Elizalde JI, García M, Gascón J, Gimeno $\mathrm{F}$, et al. Diagnosis, mangement and treatment of chronic Chagas' gastrointestinal disease in areas where Trypanosoma cruzi infection is not endemic. Gastroenterol Hepatol 2010;33:191-200.

18. Basile L, Jansà JM, Carlier Y, Salamanca DD, Angheben A, Bartoloni A, et al. Chagas disease in European countries: the challenge of a surveillance system. Euro Surveill. 2011 Sep 15;16:19968.

19. Valerio L Roure $S$, Sabrià $M$, Balanzó X, Vallès $X$, Serés L. Clinical, electrocardiographic and echocardiographic abnormalities in Latin American migrants with newly diagnosed Chagas disease 2005-2009, BarceIona, Spain. Euro Surveill. 2011;16:1997.

20. Carod-Artal FJ, Gascon J. Chagas disease and stroke. Lancet Neurol. 2010;9:533-42.
21. Pinazo MJ, Espinosa G, Cortes-Lletget C, Posada E, Aldasoro E, Oliveira I, et al. Immnosupression and Chagas disease: a managemnt challenge. PLoS Negl Trop Dis. 2013; 7:e1965.

22. Schijman AG, Altcheh J, Burgos JM, Biancardi M, Bisio $M$, Levin MJ, et al. Aetiological treatment of congenital Chagas' disease diagnosed and monitored by the polymerase chain reaction. J Antimicrob Chemother. 2003;52:441-9.

23. Oliveira I, Torrico F, Muñoz J, Gascon J. Congenital transmission of Chagas disease: a clinical approach. Expert Rev Anti Infect Ther. 2010;8:945-56.

24. Viotti R, de Noya BA, Araujo-Jorge T, Grijalva MJ, Guhl F, López MC, et al. Towards a paradigm shift in the treatment of chronic Chagas disease. Antimicrob Agents Chemother. 2014;58:635-9.

25. Viotti R, Vigliano C, Alvarez MG, Lococo B, Petti $M$, Bertocchi $G$, et al. Impact of aetiological treatment on conventional and multiplex serology in chronic Chagas disease. PLoS Negl Trop Dis. 2011;5:e1314

26. Matinero E, Roca C, Escribà JM, Bayó J, de la Fuente $S$, Grupo de estudio Chagas-Clot. Conocimiento de la existencia de la enfermedad de Chagas por inmigrantes originarios de centro y Suramérica continental. Enf Emerg. 2010,12:145-9.

27. Bern C, Montgomery SP, Herwaldt BL, Rassi A, MarinNeto JA, Dantas RO, et al. Evaluation and treatment of Chagas disease in the United States: a systematic Review. JAMA. 2007;298:2171-81.

28. González-Tomé MI, Rivera M, Camaño I, Norman F, Flores-Chávez M, Rodríguez-Gómez L, et al. Recomendaciones para el diagnóstico, seguimiento y tratamiento de la embarazada y el niño con enfermedad de Chagas. Enferm Infecc Microbiol Clin. 2013; 3:535-42. 